Präv Gesundheitsf 2014 · 9:47-51

DOI 10.1007/s11553-013-0417-y

Online publiziert: 19. November 2013

(c) Springer-Verlag Berlin Heidelberg 2013

Suzanne Lischer · Jörg Häfeli • Simone Villiger

Hochschule Luzern - Soziale Arbeit, Luzern, Schweiz

\title{
Vulnerable Personengruppen im Glücksspielbereich
}

ein problematisches Spielverhalten hat respektive gefährdet ist, ein solches zu entwickeln. Entsprechend liefern die Sperrdaten aus den Schweizer Casinos wichtige Hinweise in Bezug auf die Population glücksspielsuchtgefährdeter Personen(gruppen).

Das Erkenntnisinteresse der vorliegenden Untersuchung zielt auf die Prüfung der Frage ab, ob in der Population an gesperrten Spielern bestimmte Personengruppen besonders häufig vertreten sind und mit welchen Maßnahmen der erhöhten Problemlast begegnet werden kann.

\section{Studiendesign und Untersuchungsmethoden}

Das Forschungsprojekt besteht aus zwei separaten Teilstudien, welche nachfolgend vorgestellt werden. Es wurde ein gemischt methodischer Ansatz gewählt, bei dem sowohl qualitative wie auch quantitative Erhebungsmethoden angewendet wurden.

\section{Auswertung der Sperrdaten}

Ein Schweizer Casino hat gemäß Art. 22 Abs. 1 des Spielbankengesetztes (SBG) Personen vom Spielbetrieb auszusperren, von denen es aufgrund eigener Wahrnehmungen in der Spielbank oder aufgrund von Meldungen Dritter weiß oder annehmen muss, dass sie a) überschuldet sind oder ihren finanziellen Verpflichtungen nicht nachkommen oder b) Spieleinsätze riskieren, die in keinem Verhältnis zu ihrem Einkommen und ihrem Vermögen stehen (angeordnete Spielsperre). Ferner haben die Gäste gemäß Art. 22 Abs. 4 SBG die Möglichkeit, selber eine Spielsperre zu beantragen (freiwillige Spielsperre [12]).
Die Eintrittskontrollen in den Schweizer Casinos werden mit Hilfe der Sperrdatenbank SESAM durchgeführt. Das System erfasst sämtliche Personen, denen entweder eine Spielsperre auferlegt wurde oder die selbst eine Spielsperre beantragt haben. Damit wird die Zahl der gesperrten Spieler in der Schweiz systematisch und zuverlässig erhoben. Erfasst werden die folgenden Angaben: Name, Alter, Wohnsitz und Nationalität. Die Datenbank SESAM macht indessen keine Angaben darüber, ob es sich um eine freiwillige oder um eine angeordnete Spielsperre handelt. Nicht registriert wird außerdem das Geschlecht der gesperrten Person.

Im Rahmen der hier vorliegenden Untersuchung gibt die statistische Auswertung der Sperrdaten Aufschluss über die Gruppe derjenigen Personen, welche in den Schweizer Casinos gesperrt sind. Geprüft wird die Frage, wie hoch der Anteil der gesperrten Spieler ist, die einen Schweizer Wohnsitz haben, jedoch nicht Schweizer Staatsangehörige sind und ob es innerhalb von dieser Gruppe Nationalitäten gibt, die besonders häufig vertreten sind. Indem das Alter der gesperrten Personen ausgewertet worden ist, wird zudem ein weiteres soziodemographisches Merkmal der Angehörigen dieser Gruppe berücksichtigt.

Am 1. Januar 2012 waren in der Schweiz insgesamt 30.463 Personen gesperrt. Davon haben 22.146 Personen ihren Wohnsitz in der Schweiz (72,7\%); 8317 Personen wohnen im Ausland (27,3\%). Diese Sperrdaten waren bisher für Forschungsprojekte nicht zugänglich. Für die vorliegende Untersuchung stand erstmalig ein Teil dieser Daten zur Verfügung. Es handelt sich um die 4353 Sperrdaten des Casinos Pfäffikon, des Casinos Schaffhausen sowie des Grand Casinos St. Gallen. 


\section{Prävention/Gesundheitsförderung}

\begin{tabular}{|ll}
\hline Tab. 1 Gesprächsteilnehmende & $n$ \\
\hline Kontext der Gesprächsteilnehmenden & 10 \\
\hline Fachpersonen von Migrationsfachstellen & 1 \\
\hline Mediatorinnen von FemmesTISCHEa & 1 \\
\hline Chefin Abteilung Untersuchungen Eidgenössische Spielbankenkommission ${ }^{\text {b }}$ & 1 \\
\hline Organisator von Poker-Turnieren & 1 \\
\hline Organisator von illegalem Glücksspiel & 3 \\
\hline Privatpersonen & 17 \\
\hline Total & $\begin{array}{l}\text { aDas Projekt „FemmesTISCHE“ ist ein schweizerisches Netzwerk, das seit mehr als 10 Jahren weiterentwickelt } \\
\text { wird. Die Projektidee verbindet den geselligen Aspekt von „Tupperware-Parties“ mit den Themen Gesundheit } \\
\text { und Prävention für Migranten } \\
\text { bDie Eidgenössische Spielbankenkommission (ESBK) überwacht die Einhaltung der Vorschriften der Gesetz- } \\
\text { gebung über Glücksspiele und Spielbanken in der Schweiz und vollzieht diese }\end{array}$ \\
\hline
\end{tabular}

Tab. 2 Herkunft der gesperrten Spieler

\begin{tabular}{lll}
\hline Herkunft der gesperrten Spieler & $n$ & $\%$ \\
\hline $\begin{array}{l}\text { Ehemaliges Jugoslawien (Bosnien-Herzegowina, Mazedonien, Mon- } \\
\text { tenegro, ehem. Jugoslawien, Kosovo, Kroatien, Slowenien, Serbien) }\end{array}$ & 1.044 & 41,8 \\
\hline Türkei & 402 & 16,1 \\
\hline Italien & 273 & 10,9 \\
\hline Südostasien (Laos, Kambodscha, Philippinen, Vietnam, Thailand) & 137 & 5,5 \\
\hline Andere & 641 & 25,7 \\
\hline Gesamt & 2.497 & 100 \\
\hline
\end{tabular}

\section{Identifikation von vulnerablen Gruppen im Glücksspielbereich}

Die Leitfadengespräche zielen darauf $a b$, die Befunde der statistischen Auswertung der Sperrdaten mit der Einschätzung von Experten, welche mit der Glücksspielsuchtproblematik vertraut sind, zu ergänzen. Die verschiedenen Gesprächsteilnehmenden wurden mit Hilfe der sog. „Nominationstechnik“ gewonnen, d. h. von den befragten Personen wurden weitere Personen angegeben, die besondere Kenntnisse zu dem Themenbereich Glücksspielsucht mitbringen. Insgesamt haben 17 Personen an den Leitfadengesprächen mitgewirkt (• Tab. 1).

Die Leitfadengespräche mit Experten von Migrationsfachstellen und aus dem Glücksspielbereich sowie mit Privatpersonen (u. a. zwei Angehörige von Personen mit einem problematischen Spielverhalten) gingen der Frage nach, inwiefern bestimmte Bevölkerungsgruppen mit Migrationshintergrund identifiziert werden können, die stärker von glücksspielsuchtbezogenen Problemen betroffen sind. Außerdem wurde danach gefragt, welche Glücksspielformen die jeweiligen Bevölkerungsgruppen spie- len, wo sie diese spielen und ob spezifische glücksspielsuchtbezogene Probleme ausgemacht werden können. Ferner wurde die Frage geprüft, mit welchen präventiven Angeboten dieser spezifischen Probelast begegnet werden kann, inwiefern diese Hilfsangebote bei den betroffenen Personengruppen bekannt sind und in welchem Umfang sie diese nutzen.

\section{Ergebnisse aus der Auswertung der Sperrdaten}

Die Daten von 4353 Spielsperren, die dem Forschungsprojekt zur Verfügung stehen, betreffen in 4038 Fällen $(92,8 \%)$ Personen, die in der Schweiz wohnhaft sind, und in 315 Fällen (7,2\%) Personen, die im Ausland leben. Bei den 4038 gesperrten Personen, welche in der Schweiz ihren Wohnsitz haben, handelt es sich bei insgesamt 2497 (61,8\%) um ausländische Staatsangehörige mit Wohnsitz in der Schweiz. Dies entspricht einem Anteil von 57,3\% an den insgesamt zur Verfügung stehenden Spielsperren. Sie stellen die Stichprobe der ersten Untersuchung dar.
Tab. 3 Alter der gesperrten Spieler

\begin{tabular}{|lll|}
\hline Altersgruppe (Jahre) & $\boldsymbol{n}$ & $\%$ \\
\hline $18-25$ & 154 & 6,2 \\
\hline $26-35$ & 862 & 34,5 \\
\hline $36-45$ & 822 & 32,9 \\
\hline $46-55$ & 457 & 18,3 \\
\hline $56-65$ & 156 & 6,2 \\
\hline$\geq 66$ & 45 & 1,8 \\
\hline Unbekannt & 1 & $<0,0$ \\
\hline Gesamt & 2.497 & 100,0 \\
\hline
\end{tabular}

Insgesamt sind bei den gesperrten Spielern 75 verschiedene Nationalitäten vertreten. - Tab. 2 liefert einen Überblick. Von den insgesamt 2497 Spielsperren betreffen 1044 Personen aus dem ehemaligen Jugoslawien, 402 Personen aus der Türkei, 273 Personen aus Italien und 137 Personen aus Südostasien; 641 Personen haben eine Nationalität, die nicht häufig vertreten war und deshalb unter der Kategorie „Andere“ zusammengefasst wurde.

In der Auswertung berücksichtigt wurde zudem das Alter der gesperrten Personen. Aus der Tab. 3 ist ersichtlich, dass die Altersgruppe der 26- bis 35-Jährigen mit $34,5 \%$ am häufigsten vertreten ist, gefolgt von der Altersgruppe der 36bis 45-Jährigen mit 32,9\%. Die restlichen Altersgruppen sind mit $<20 \%$ vertreten.

Die beiden oben angeführten Tabellen liefern wichtige Hinweise in Bezug auf die Population glücksspielsuchtgefährdeter Personen(gruppen). Allerdings muss bei der Interpretation beachtet werden, dass die Tatsache einer Spielsperre nicht automatisch auf eine erhöhte Gefährdungslage der Person schließen lässt. Die Gründe für eine Spielsperre sind sehr vielfältig. Ein Spieler hat z. B. die Möglichkeit, eine sog. „präventive freiwillige Spielsperre “ $\mathrm{zu}$ beantragen.

Als weitere Einschränkung gilt es zudem zu beachten, dass diese Daten in eine Relation zu der Besucherpopulation in Schweizer Casinos gebracht werden müssten. Dazu fehlen jedoch die entsprechenden Daten. Erst die Relation der oben angeführten soziodemographischen Daten der gesperrten Personen mit denjenigen der allgemeinen Besucherpopulation würde entsprechende Auffälligkeiten betreffend allfällig erhöhter Gefährdung bei einzelnen Spielergruppen erlauben. 


\section{Ergebnisse aus den Leitfadengesprächen}

Den Beobachtungen der Gesprächsteilnehmenden zufolge lassen sich verschiedene Bevölkerungsgruppen mit Migrationshintergrund identifizieren, welche bezüglich glücksspielsuchtspezifischer Probleme von einer erhöhten Problemlast betroffen sind. Demnach sind Personen aus dem Raum des ehemaligen Jugoslawien, der Türkei, aus Italien, Spanien und Portugal besonders betroffen. Auch Personen aus dem südostasiatischen Raum werden als besonders vulnerable Gruppe wahrgenommen. Die Einschätzungen der Experten stimmen somit mit den quantitativen Auswertungen der Sperrdaten größtenteils überein.

Auf die Frage nach spezifischen glücksspielsuchtbezogenen Problemen für diese Personengruppen betonten mehrere Gesprächsteilnehmende, dass die Illusion des „schnellen“ Geldes als bedeutender Risikofaktor zu gewichten ist. Nun liegt es in der Natur der Sache, dass diese Illusion in der Regel nicht zu einer Einkommensvermehrung führt, sondern zu einem Einnahmeverlust. Entsprechend zeigen sich die glücksspielsuchtspezifischen Probleme bei den Betroffenen v. a. in Form von finanziellen Schwierigkeiten. Neben den glücksspielsuchtbezogenen Problemen der Spieler selbst darf die problematische Situation der Angehörigen nicht außer Acht gelassen werden. So können glücksspielsuchtspezifische Probleme zu Trennungen beziehungsweise zerrütteten $\mathrm{Fa}$ milienverhältnissen führen. Bei den Angehörigen von Glücksspielsüchtigen stellt sich zudem oftmals das Problem der Koabhängigkeit des Partners oder der Partnerin.

In Bezug auf institutionelle Hilfe von Beratungs- oder Behandlungsstellen gilt es als gesicherte Erkenntnis, dass nur ein kleiner Anteil von Personen mit Glücksspielproblemen institutionelle Hilfe von Beratungs- oder Behandlungsstellen in Anspruch nimmt [3]. Die Gesprächsteilnehmenden äußerten die Einschätzung, dass Beratungs- und Behandlungsangebote für Personen mit glücksspielsuchtspezifischen Problemen speziell bei Migranten zum einen wenig bekannt sind und zum anderen - sofern bekannt -

Präv Gesundheitsf 2014 · 9:47-51 DOI 10.1007/s11553-013-0417-y

(c) Springer-Verlag Berlin Heidelberg 2013

\section{S. Lischer · J. Häfeli · S. Villiger \\ Vulnerable Personengruppen im Glücksspielbereich}

Zusammenfassung

Hintergrund. Eine Voraussetzung für eine ressourcenoptimierte Präventionspraxis ist die Kenntnis darüber, welche Faktoren die Wahrscheinlichkeit eines problematischen Spielverhaltens erhöhen.

Methodik. Neben der Auswertung von Sperrdaten aus Schweizerischen Casinos wurden leitfadengestützte Interviews mit Experten von Beratungsstellen sowie mit Privatpersonen durchgeführt.

Ergebnis. Im Rahmen der Ergebnisdarstellung wird mit dem Alter und dem Migrationshintergrund auf zwei Risikofaktoren verwiesen. Die besondere Vulnerabilität letzterer

\begin{abstract}
Personengruppe lässt sich indessen weniger auf deren Herkunft als auf die situativen Bedingungen der Migration zurückführen.

Schlussfolgerung. Es besteht Bedarf an Präventionsmaßnahmen, welche sich gezielt an die vulnerablen Personengruppen richten. Mit Blick auf die hohe Zahl von kumulierten Spielsperren in der Schweiz sind die Personen, die von den Schweizer Casinos gesperrt sind, im Besonderen zu berücksichtigen.
\end{abstract}

\section{Schlüsselwörter}

Glücksspielsucht · Spielsperren · Prävention . Behandlung · Vulnerable Gruppen

\section{Vulnerable population groups in the field of gambling}

\section{Abstract}

Background. An important precondition for a resource optimized prevention practice is to know the factors, which raise the probability of a problematic gambling behaviour. Method. Besides the analysis of the bans from Swiss Casinos, interviews with experts from counselling centres as well as with private persons were conducted.

Results. The results show, that age and migration background are two risk factors. It is emphasized, that this special vulnerability of the last-mentioned population group can be traced back to the situative conditions rather than to their origin.
Conclusion. There is a necessity for preventive measures, which are directed at vulnerable population groups. With the high numbers of cumulated bans in Switzerland in mind, special thoughts have to be given to measures directed at those people, who are banned from Swiss Casinos.

\section{Keywords}

Gambling addiction - Bans · Prevention .

Treatment $\cdot$ Vulnerable groups kaum genutzt werden. Entsprechend drängt es sich auf, mögliche Zugangshürden abzubauen. Daneben gilt es aber auch, alternative Kanäle zur Vermittlung von präventiven Botschaften zu nutzen. Im Rahmen der Leitfadengespräche wiesen die Experten auf die folgenden Vorschläge hin:

- Großes Potenzial für die Kommunikation von präventiven Botschaften sehen die befragten Fachpersonen im Einbezug von interkulturellen Vermittlern oder in Fachpersonen mit Migrationshintergrund. Es gilt, institutionalisierte Konzepte zu berücksichtigen, welche auf den Prinzipien des Empowerments aufbauen.

- Eine weitere Möglichkeit, präventive Botschaften zu vermitteln, wird in der Vermittlung von präventiven
Botschaften via Medien (Print, Fernsehen und Radio) gesehen. Auf diese Weise erhält man eine hohe Reichweite bei den angestrebten Zielgruppen.

- Da sich glücksspielspezifische Probleme in der Regel erst im Zusammenhang mit finanziellen Schwierigkeiten zeigen, ist es wichtig, dass Fachpersonen aus dem externen Versorgungssystem bezüglich glücksspielsuchtspezifischer Probleme und anderer Verhaltenssüchte geschult sein müssen.

\section{Diskussion}

Die Glücksspielforschung in der Schweiz steht noch ganz am Anfang. Im Rahmen dieses Forschungsprojekts konnten ver- 
schiedene Aspekte der Glücksspielsucht behandelt werden. Aus den Befunden geht jedoch auch hervor, dass Vertiefungen notwendig sind.

\section{Bestimme Personengruppen mit erhöhter Vulnerabilität}

Es hat sich in beiden Teilen der Untersuchung gezeigt, dass sich Bevölkerungsgruppen identifizieren lassen, die besonders häufig von einer Spielsperre betroffen sind. Es sind dies Personen im Alter von 26-45 Jahre. Außerdem sind bei den gesperrten Spielern Personen mit Migrationshintergrund überproportional vertreten. Die Schlussfolgerung, dass Migranten grundsätzlich von einer erhöhten Glücksspielsuchtprävalenz betroffen sind, wäre aber voreilig. Die Experten führen die Hypothese an, dass die besondere Vulnerabilität dieser Personengruppen weniger auf deren Herkunft als auf die besonderen Lebensumstände, welche typisch für Migranten sein können ( $\mathrm{Zu}$ gangshürden zu Institutionen, Chancenungleichheit in Ausbildung und Beruf, enge Wohnverhältnisse, etc.) zurückzuführen ist.

- Situative Bedingungen der Migration als Risikofaktor für glücksspielsuchtspezifische Probleme

Diese Einschätzung wird auch von Fachpersonen aus Deutschland geteilt [11]. Entsprechend gilt es, spezifische Präventions- und Beratungsangebote zu entwickeln, die den besonderen Bedürfnissen dieser vulnerablen Gruppen gerecht werden. Die Notwendigkeit, entsprechende Angebote in verschiedenen Sprachen anzubieten, wird grundsätzlich nicht in Frage gestellt, da gerade bei Problemspielern mit Migrationshintergrund die Sprache als zusätzliche Barriere für die Inanspruchnahme von Hilfsangeboten dazukommen kann [9].

Grundsätzlich gilt es jedoch zu beachten, dass spezifische Präventions- und Beratungsangebote, die nur die Herkunft der Adressaten berücksichtigen, Gefahr laufen, der Heterogenität einzelner Gruppen und deren individuellen Bedürfnissen nicht gerecht zu werden. Eine Definition der Zielgruppe nur anhand der Herkunft ignoriert sozioökonomische Fakto- ren und Einflüsse wie Alter, Geschlecht und Aufenthaltsdauer im Residenzland, welche die Lebenssituation von Personen mit Migrationshintergrund stark bestimmen. Auch die heterogenen persönlichen Voraussetzungen (z. B. Religion, Migrationserfahrung) und die Bedingungen im Herkunftsland (soziokulturelle Prägungen, Erfahrungen mit dem Gesundheitssystem etc.) werden so zu wenig berücksichtigt. Es darf nicht davon ausgegangen werden, dass Personen mit Migrationshintergrund die gleichen Bedürfnisse haben, nur weil sie aus demselben Herkunftsland stammen [8].

\section{Effektivität der Spielsperre}

Obwohl die Spielsperre als Maßnahme des Spielerschutzes weltweit zunehmend in verschiedenen (Glücksspiel-)Marktsegmenten zum Einsatz kommt, fehlt bislang eine hinreichende und differenzierte Begleitforschung zur Evaluierung ihres Nutzens [6]. Beispielsweise beziehen sich die wenigen Forschungsbefunde ohne Ausnahme auf die Selbstsperre, wissenschaftlich fundierte Daten zur angeordneten Spielsperren finden sich indessen nicht (ebd.). Wissenschaftliche Befunde hierzu sind also notwendig, auch im Hinblick auf die Weiterentwicklung der Sozialkonzepte der Glücksspielanbieter und für die Ausgestaltung präventiver Maßnahmen im Allgemeinen.

- Frage nach der Effektivität der Spielsperren

Personen mit einem exzessiven Spielverhalten nehmen Glücksspiele als komplexe Einheit wahr und differenzieren nicht mehr zwischen legalem und illegalem Glücksspiel. Wenn die eine Spielmöglichkeit eingeschränkt ist, dann wird auf eine andere ausgewichen. Die Interventionsformen unterscheiden sich dagegen je nachdem, ob es sich um Casinospiele oder um Formen des illegalen Glücksspiels handelt. Grundsätzlich können Personen mit einem problematischen Spielverhalten vom Glücksspiel in den Casinos ausgeschlossen werden. Als Ausweichmöglichkeit bietet sich jedoch das Spiel in den grenznahen Casinos an [4]. Für das illegale Glücksspiel bestehen naturgemäß weniger Interventionsmöglichkeiten.
Den Betroffenen oder deren Angehörigen bleibt oft nur die Möglichkeit einer Anzeige gegen den Veranstalter. Es kann davon ausgegangen werden, dass die in der Schweiz erzielten Umsätze aus dem illegalen Glücksspiel und über die aus dem Ausland operierenden Remote-Anbieter höher sind, als diejenigen aus dem legalen Glücksspiel (konzessionierte Casinos und Lotterien und Wetten). Ebenso darf vermutet werden, dass vulnerable Personengruppen eher auf diese Angebote ausweichen oder eher davon angesprochen werden. Neben der Frage nach der Effektivität der Spielsperren als präventive Maßnahme stellt sich somit auch die Frage, inwiefern ein Ausschluss den Nährboden für illegales Glücksspiel bietet. Neben der Verstärkung der Kontrollen von illegalen Glücksspielanbietern durch die Justiz sind daher Präventionsmaßnahmen, die sich gezielt an gesperrte Spieler richten, auszubauen. Dies kann die Abgabe von Informationsmaterial sein, das Auskunft über die Rechtslage sowie die Risiken des illegalen Glücksspiels vermittelt. Es sollte aber auch darüber informiert werden, wie man als Betroffener gegen entsprechende Anbieter vorgehen kann.

\section{Fazit für die Praxis}

Aus den Ergebnissen kann die vorläufige Schlussfolgerung gezogen werden, dass es in der Schweiz bestimmte Personengruppen mit einer erhöhten Vulnerabilität für glücksspielsuchtbezogene Probleme gibt. Entsprechend stellt sich die Frage nach der Ausgestaltung von präventiven Maßnahmen, die sich gezielt an die entsprechenden Personengruppen richten. Mit Blick auf die hohe Zahl von kumulierten Spielsperren in der Schweiz stellt sich im Speziellen die Frage nach präventiven Maßnahmen für diejenigen Spieler, die von den Schweizer Casinos gesperrt sind.

\section{Korrespondenzadresse

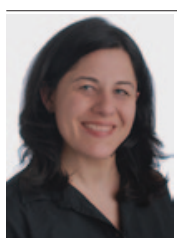 \\ lic.rer.soc. S. Lischer Hochschule Luzern - Soziale Arbeit Werftestrasse 1 CH-6002 Luzern suzanne.lischer@hslu.ch}




\section{Einhaltung ethischer Richtlinien}

Interessenkonflikt. S. Lischer, J. Häfeli und S. Villiger geben an, dass kein Interessenkonflikt besteht. Die vorliegende Studie wurde hauptsächlich durch das Mandat Interkantonales Programm Glücksspielsuchtprävention der Nordwest- und Innerschweiz, vertreten durch Sucht Schweiz finanziert. Die korrespondierenden Autoren versichern, dass sie jederzeit unabhängig in der Wahl der Forschungsfragen, der Forschungsmethoden und der Darstellung der Resultate waren.

\section{Literatur}

1. Bundeszentrale für Gesundheitliche Aufklärung (Hrsg) (2012) Glückspielverhalten und Glücksspielsucht in Deutschland. Ergebnisse aus drei repräsentativen Bevölkerungsbefragungen 2007, 2009 und 2011. Bundeszentrale für Gesundheitliche Aufklärung, Köln

2. Hayer T, Meyer G (2010) Glücksspielsucht: Problemausmaß, Risikofaktoren und Spielerschutz. Public Health Forum 18(67):27-29

3. Kilian K, Fritschi T, Oesch T et al (2009) Soziale Kosten des Glücksspiels in Casinos. Studie zur Erfassung der durch die Schweizer Casinos verursachten sozialen Kosten. Im Auftrag der Eidgenössischen Spielbankenkommission ESBK, Bern

4. Lischer S, Häfeli J (2012) Spielerschutzmaßnahmen im Rahmen einer kohärenten Glücksspielpolitik. In: Kirch W, Hoffmann T, Pfaff H (Hrsg) Prävention und Versorgung. Thieme, Stuttgart, S 165-174

5. Meyer G, Bachmann M (2005) Spielsucht - Ursachen und Therapie. Springer, Berlin Heidelberg New York

6. Meyer G, Hayer T (2010) Die Effektivität der Spielsperre als Maßnahme des Spielerschutzes - Eine empirische Untersuchung von gesperrten Spielern. Lang, Frankfurt a. M.

7. Meyer C, Rumpf H-J, Kreuzer A et al. (2011) Pathologisches Glücksspielen und Epidemiologie (PAGE): Entstehung, Komorbidität, Remission und Behandlung. Endbericht. Greifswald, Lübeck

8. Pfluger T, Biedermann A, Salis Gross C (2009) Transkulturelle Prävention und Gesundheitsförderung in der Schweiz. http.//www.transpraev.ch. Zugegriffen: 12. Dez 2012

9. Sabbioni M, Salis Gross C (2006) Die migrationsspezifische Anamnese. In: Van Eeuwijk P, Obrist B (Hrsg) Vulnerabilität, Migration und Altern. Medizinethnologische Ansätze im Spannungsfeld von Theorie und Praxis. Seismo, Zürich S 166-201

10. Salis Gross C, Moser C, Zuppinger B, Hatz C (1997) Die Arzt-Patienten Interaktion aus der Sicht von MigrantInnen: Vorschläge für die ärztliche Praxis. Schweizerische Rundschau für Medizin (PRAXIS). Sondernummer "Migration und Gesundheit ${ }^{\prime \prime}$ 86:887-894

11. Tuncay M (2010) Glücksspiel bei Migranten aus dem orientalischen Kulturraum. http://www.konturen.de. Zugegriffen: 14. Jan 2013

\section{Rechtliches Dokument}

12. Bundesgesetz über Glücksspiele und Spielbanken (Spielbankengesetz, SBG) vom 18. Dezember 1998 (Stand am 27. Dezember 2006). http://www.admin.ch. Zugegriffen: 17. Dez 2012 